\title{
INTERVENCIONES URBANAS: EXPERIENCIA ACADÉMICA EN EL DESARROLLO DE PROYECTOS ESTRATÉGICOS
}

José Alejandro Gómez Suárez.*

Edwin Balcacer, Gustavo Rey, Gonzalo Valenzuela, Nathalia Parra, Gustavo Lizcano, Ómar Méndez, Carlos a Valencia, Jénnifer Martínez, Isabel Quiñónez, María B. Fuentes, Jenny P. Correa, Pedro Ardila, Nicolás Romero.***

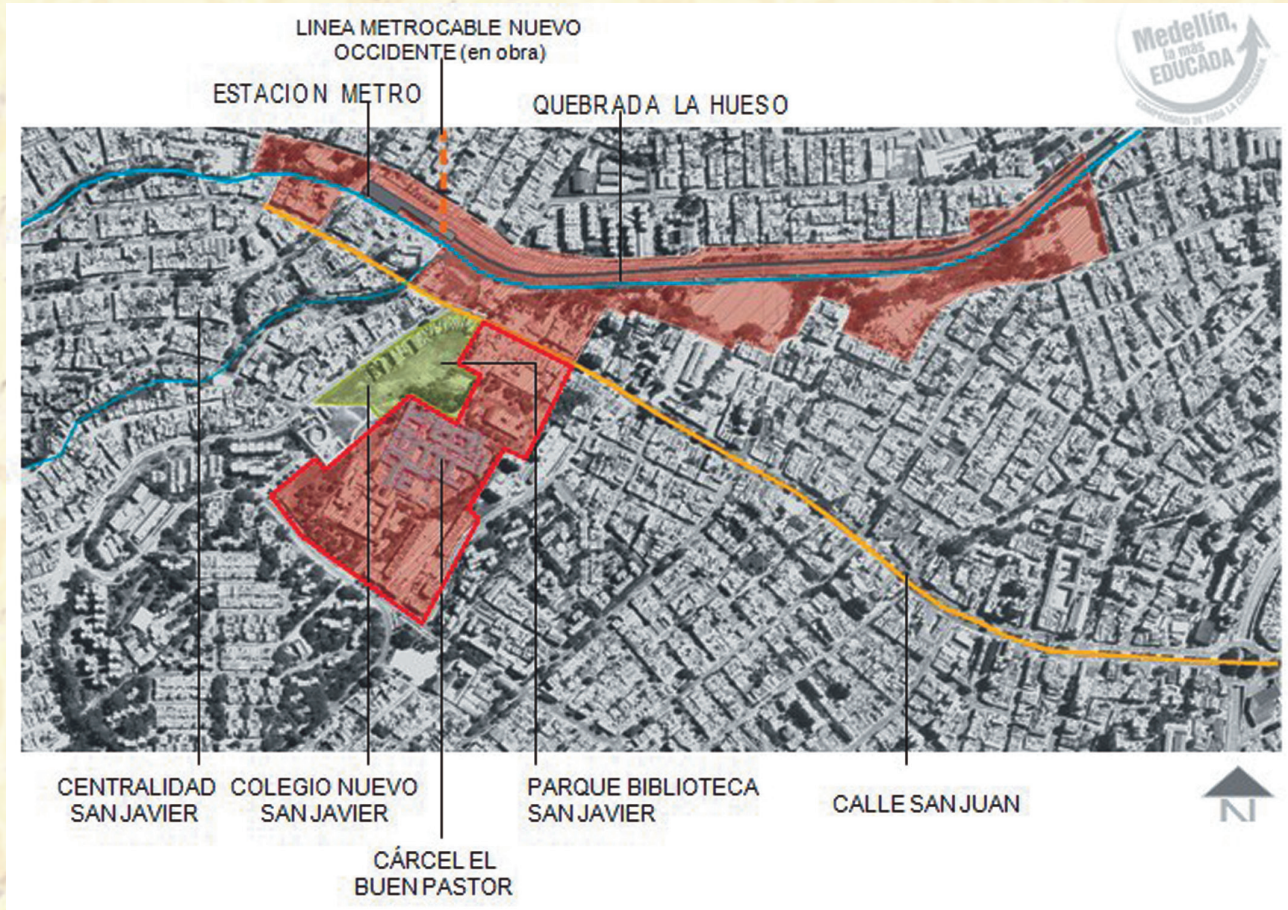

\section{RESUMEN}

El desarrollo de ejercicios de intervención urbano arquitectónica desarrollado por alumnos y docentes de Taller IX durante el primer semestre de 2007 en ciertos sectores de la ciudad de Medellín, con motivo de la convocatoria realizada por la Alcaldía Municipal y la Empresa de Desarrollo Urbano - EDU, se ha constituido en la base para el establecimiento de puntos de contacto entre la rigurosidad de la interpretación y reflexión académica, con los reales procesos de intervención en la ciudad, se abre un espacio de reconocimiento de la actividad académica desarrollada en el ámbito regional y proyectada en el contexto nacional, y experimenta una forma de acercamiento a la realidad cotidiana de la ciudad y a las más recientes formas de gestión y planificación territorial desarrolladas en el país.

\section{PALABRAS CLAVE}

proyecto, urbano, estratégico 


\section{URBAN INTERVENTIONS: ACADEMIC EXPERIENCE IN THE STRATEGIC PRO JECT DEVELOPMET.}

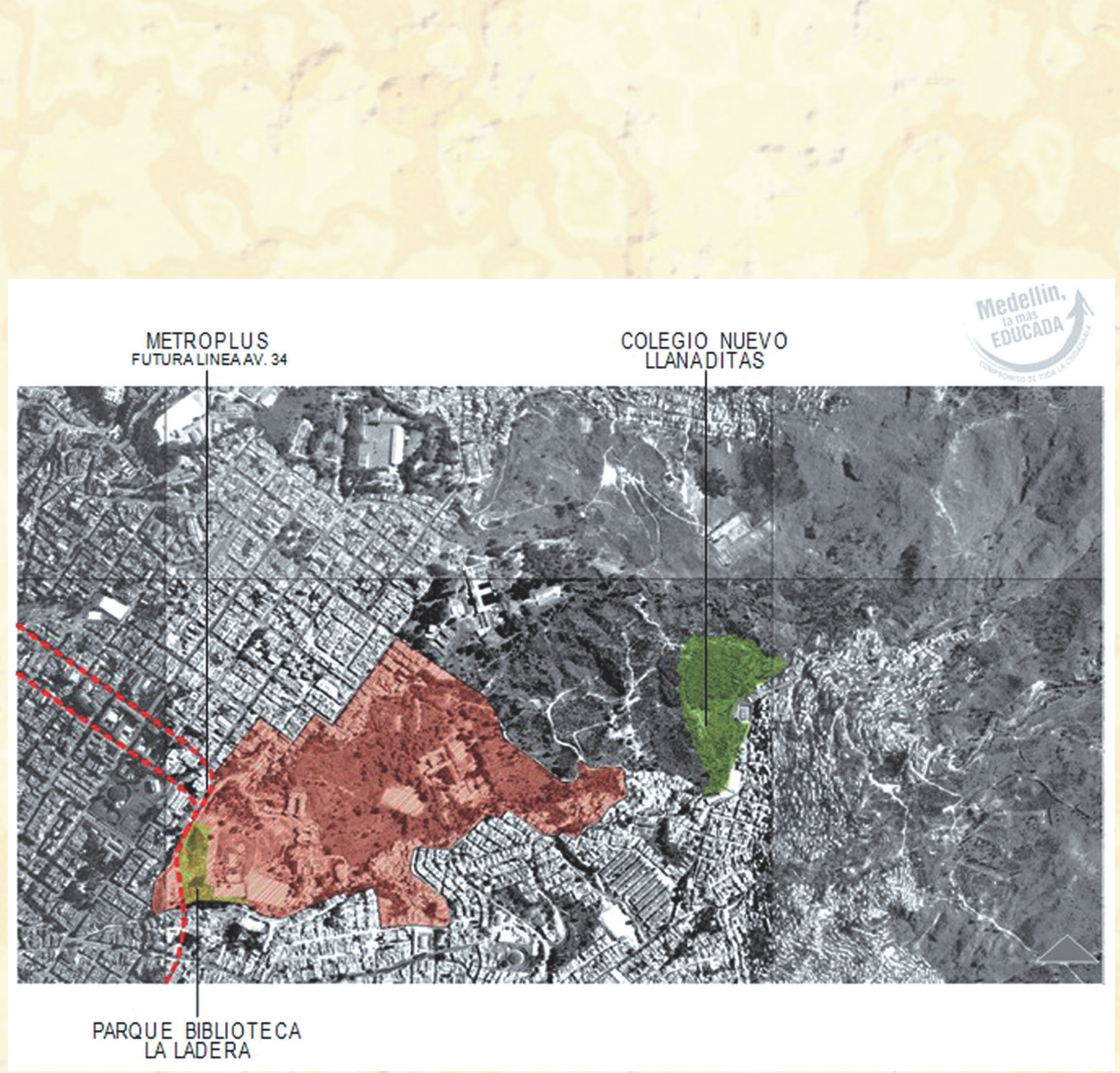

Arquitecto de la USTA, Seccional Bucaramanga; Especialista en Planificación Urbana y Regional, Universidad de Buenos Aires; Docente Universidad Santo Tomás, Seccional Bucaramanga, Facultad de Arquitectura.

** Grupo de Estudiantes Taller IX

\section{ABSTRACT}

The Academy has been leading a debate about urban interventions in the most affected areas and also the areas with a potential re-development and those with a possibility to become part of the urban fabric. The debate has been constituted as a assessment between a radical interpretation and an academic analysis taking actual urban intervention processes leaded by architectural students, the community and the strategic projects which started the urban transformation processes.

\section{KEY WORDS}

Project, urban, strategic 


\section{PROBLEMA}

A partir de la convocatoria realizada por la Alcaldía de Medellín, para participar en el “Encuentro Medellín 2007", la Facultad de Arquitectura de la Universidad Santo Tomás Seccional Bucaramanga realizó un trabajo académico durante el primer periodo de 2007 con los alumnos regulares de noveno semestre, en el marco del desarrollo de la asignatura Taller IX. El tema central del encuentro consistió en la elaboración de propuestas urbanas y arquitectónicas en cuatro sectores de la ciudad, con marcados procesos de deterioro pero con potenciales de re-desarrollo e incorporación a la estructura urbana, en los cuales fueron construidos importantes proyectos estratégicos de carácter comunitario y de espacio público.

El conocimiento, la interpretación y el análisis sobre el estado de situación de las áreas definidas previamente por la Empresa de Desarrollo Urbano de Medellín - EDU, se planteó como uno de los primeros escollos por superar, principalmente a partir de recorridos urbanos y viajes a las entrañas de la ciudad, cuyos protagonistas de excepción fueron los jóvenes estudiantes de arquitectura de las diferentes facultades, la comunidad y los mismos proyectos estratégicos que dieron inicio a los procesos de transformación urbana aún en curso.

El "Encuentro Medellín 2007" planteado desde su inicio como una fiesta de urbanismo y arquitectura, de urbanidad y participación comunitaria en los barrios de la ciudad, resaltó la imperativa necesidad de involucrar a la academia en los procesos de intervención en las estructuras territoriales, por lo que el riguroso proceso de interpretación y reflexión que desde el Taller de diseño se realizó, se constituyó en el fundamento para la articulación y la generación de puntos de contacto entre todos los actores intervinientes.

\section{OBJETIVOS}

El propósito de la convocatoria consistió en reunir a un grupo importante de facultades de arquitectura de diferentes ciudades de Colombia para que, a través de un trabajo académico, elaborasen propuestas de intervención en ciertos sectores de la ciudad de Medellín, las cuales serían confrontadas en un gran encuentro de reflexión académica, debate urbano y de recorridos de estudio por toda la ciudad.

El espacio abierto para la reflexión se orientó hacia la valoración de cuatro ejes temáticos fundamentales', a partir de los cuales se desarrolló el debate en torno al mejor modelo de intervención para cada sector, según sus características propias:

Alejandro Echeverri Restrepo, Encuentro Medellín 2007, EDU, 2007

- El relieve geográfico y el valor singular del paisaje natural del Valle deben pasar de ser un grave problema, a convertirse en zonas de oportunidad y de valor singular de nuestra identidad urbana.

- Recuperar el valor esencial de la Calle Urbana, la calle para la gente. Se busca calidad urbana y actividad en las calles y paseos representativos de la ciudad y los barrios.

- El edificio público como nodo de actividades con servicios integrales de calidad. El Espacio Público y el Edificio Público como lugares para el reencuentro ciudadano.

- Los proyectos de vivienda como solución para recomponer los tejidos urbanos de los barrios e incorporar otros usos para garantizar la actividad urbana. 


\section{JUSTIFICACIÓN}

Ante el complejo panorama presente y futuro de una ciudad marcada por serios problemas de inequidad social, de segregación espacial y de desequilibrios territoriales, la Administración Municipal de Medellín en cabeza de la Empresa de Desarrollo Urbano - EDU, desarrolló una ágil política de intervención urbana a través de la implementación de estrategias en materia de gestión y planificación, producto de una visión territorial que resalta el aprovechamiento de las ventajas comparativas de los escenarios físicos y sociales, para un mejor uso y aprovechamiento del territorio.

Con la implementación del modelo de intervención territorial, se dio inicio al pago de la deuda social con la población menos favorecida, a partir del desarrollo de 5 familias de programas y proyectos ${ }^{2}$ :

- Planear para no improvisar: Revisión del Plan de Ordenamiento Territorial actual y elaboración de todos los planes de escala intermedia pendientes. Elaboración del Plan Especial del Poblado, Plan Especial del Centro; Plan Especial de Patrimonio y el Plan Especial de Espacio Público y Equipamientos, entre otros.

- Programa de Parques Bibliotecas y Equipamientos Educativos para dignificar los barrios: fortalecimiento de las centralidades barriales con grandes equipamientos educativos, culturales y servicios integrales de calidad (5 Parques Biblioteca, 10 nuevos colegios de calidad, renovación de 132 sedes educativas, programa de los Centros de Desarrollo Empresarial (CEDEZOS), el Parque Explora de ciencia y tecnología y la Renovación del Jardín Botánico, entre otros.

- Proyectos Urbanos Integrales -PUI- contra la exclusión y la desigualdad: proyectos Urbanos que incorporan todos los elementos del desarrollo de forma planeada y simultánea en un territorio definido, con la activa participación de la comunidad. Se localiza la inversión en barrios de origen marginal con problemas profundos de desigualdad: Proyecto urbano Integral - PUI Nororiental, PUI Comuna 13, Intervención Integral de Moravia.

- Vivienda Social para solucionar deudas históricas: la inversión pública en vivienda se orienta a las poblaciones de menores ingresos en situaciones críticas de habitabilidad. Se busca solucionar problemas de zonas de riesgo y hacinamiento con la reubicación de la población en nuevos barrios de expansión como la Ciudadela Nuevo Occidente.

- Plan de Paseos y Calles Emblemáticas: se busca recuperar la calidad urbana en las calles y paseos representativos de la ciudad y los barrios. Recuperar el valor esencial de la Calle Urbana, la calle para la gente. Los proyectos incorporan de forma simultánea el espacio público, la movilidad con prioridad en el transporte público y la cultura urbana de la participación ciudadana: el Paseo de Carabobo, el Paseo de Andalucía, el Paseo de la 45, la Avenida del Poblado, la Avenida de San Juan, la Avenida Oriental, y el Sistema Metroplus, entre otros.

Bajo esta perspectiva la EDU definió cuatro sectores para ser analizados desde la academia, a partir de la vivencia experimentada por los alumnos de las distintas facultades de arquitectura, con el fin de estructurar una intervención urbana articulada basada en los proyectos estratégicos que han dado impulso al re-desarrollo de los barrios y las comunas donde se encuentran insertos:
2 Alejandro Echeverri Restrepo, Medellín Problemas y Oportunidades, EDU, 2007 
IMAGEN I. Localización de los sectores que se van a intervenir.

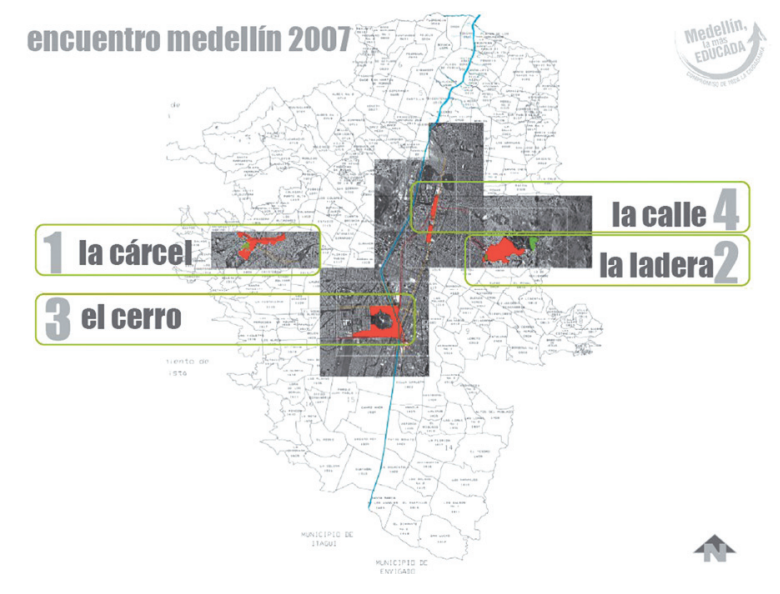

FUENTE: EDU Medellín.

- Sector LA CÁRCEL: El lugar del Proyecto Urbano está localizado en el sector centrooccidental de la ciudad, y el polígono de intervención tiene un área de 25 hectáreas. Se propone desarrollar un nuevo barrio de vivienda y servicios en el predio de la actual cárcel del Buen Pastor y redefinir el parque lineal de la Quebrada La Hueso con usos de equipamientos deportivos que salven la ruptura causada por la línea del Metro. Se busca, a través del Proyecto Urbano, resolver la relación entre: la centralidad de San Javier, la estación del Metro y estación de llegada del futuro Metrocable de Nuevo Occidente, los nuevos equipamientos como el Parque Biblioteca de San Javier y el Colegio EPM, el predio de la Cárcel del Buen Pastor y el sistema lineal de la quebrada la Hueso.

- Sector LA LADERA: el lugar del Proyecto Urbano está localizado en el sector centrooriental de la ciudad, y el polígono de intervención tiene un espacio de 37 hectáreas. Se propone consolidar un borde en la parte alta de la ciudad, a partir de complementar un vacío de ciudad por medio de nuevos barrios y un parque urbano. Se encuentra en un contexto urbano entre barrios consolidados, la presión de ocupaciones marginales, equipamientos públicos, y el límite entre el sistema natural y urbano.

- Sector EL CERRO: el lugar del Proyecto Urbano está localizado en el sector central de la ciudad, el polígono de intervención tiene una extensión de 48 hectáreas. Se propone redefinir el límite entre el Parque del Cerro Nutibara y la ciudad, con nuevos usos de servicios y públicos de escala metropolitana. Hacia la calle 30 donde se construye el nuevo corredor de Metroplus se busca la transformación del suelo industrial en nuevos usos como comercio y servicios. Hacia el barrio Fátima solucionar la relación de accesibilidad al cerro. Y hacia la calle 33, el sector de plaza mayor y el río se busca la transformación del suelo residencial y residual por equipamientos públicos metropolitanos.

- Sector LA CALLE: el polígono de intervención tiene una superficie de 10 hectáreas. Renovación urbana de la franja Carabobo-Cundinamarca, entendida como principal sistema estructurante del centro de la ciudad. La calle Carabobo ha sido desde la fundación de la ciudad, el lugar donde se han localizado los edificios públicos más representativos $y$, ha sido el eje ordenador del crecimiento del centro. Actualmente está en un proceso de recuperación urbana aunque el polígono definido para trabajarlo es un suelo urbano que presenta un alto deterioro y es poco consolidado y se busca en ella aumentar la intensidad del uso del suelo con nuevos usos y un nuevo sistema de espacios públicos secuenciales. 


\section{MARCO CONCEPTUAL}

Basada en la implementación de proyectos estratégicos como una alternativa para generar procesos ágiles de recualificación urbana, la EDU ha desarrollado una novedosa forma de gestión y planificación urbana basada en la visión de desarrollo futuro y presente de la ciudad de Medellín, a partir de la generación de escenarios de competitividad territorial que permitan alcanzar el bienestar y la equidad social.

A través de la intervención estratégica y planificada, que abarca las dimensiones de lo físico, lo social y lo institucional, la EDU ha encontrado el camino para la resolución de las problemáticas específicas de un territorio particular y con una ausencia generalizada del Estado y procura el mejoramiento de las condiciones de vida de sus habitantes.

La Gestión y la Planificación Estratégica Urbana se constituyen en los fundamentos del modelo de desarrollo de la ciudad, orientado hacia la canalización de la inversión pública según los indicadores de desarrollo humano (IDH) y de calidad de vida, en busca de la equidad socio territorial, tenida como base la intervención en el espacio público y los equipamientos, en el marco de la educación y la cultura, como lugares para el reencuentro ciudadano 3 .

\section{MATERIALES Y MÉTODOS}

Desde la perspectiva académica, el desarrollo del tema encomendado por la EDU se estructuró al interior de la Facultad de Arquitectura por parte de los docentes titulares de Taller IX Luis Ardila Cancino y José Alejandro Gómez, mediante el análisis y desarrollo de propuestas de intervención urbana, que respondiesen a las necesidades espaciales de los sectores de la ciudad previamente definidos. Para ello, se tomó como referencia el área delimitada por la EDU y su interrelación con el entorno inmediato, a través de tres escalas de trabajo:

- Reflexión conceptual sobre el contexto general del proyecto urbano (Análisis del contexto de la forma urbana y arquitectónica).

- Desarrollo del proyecto urbano en sus componentes urbano-arquitectónicos, mediante una reinterpretación del programa sugerido por el Municipio de Medellín (Propuesta de intervención urbana).

- Desarrollo de una o varias piezas arquitectónicas o proyectos de espacio público como proyectos singulares (Propuesta arquitectónica).

Así mismo se realizó un abordaje e introducción al conocimiento y aplicación de metodologías de análisis y selección de las variables más relevantes que inciden en la construcción de la ciudad y los sectores urbanos específicos, así como sus estrategias de planeamiento y gestión:

- Definición de metodología y escalas de trabajo

- Reconocimiento del sector urbano

- Elaboración de un análisis urbano e identificación de componentes

- Definición de criterios de implantación de la propuesta

- Formulación de estrategias de intervención y gestión

- Desarrollo del proyecto urbano
3 Alejandro Echeverri Restrepo, MedeIlín Problemas y Oportunidades, EDU, 2007 
Durante la fase inicial se organizaron cinco (5) grupos de trabajo con los alumnos distribuidos en cada uno de los sectores.

Igualmente, se recolectó la información básica y se desarrolló un análisis de las áreas intervenidas mediante una visita técnica a la ciudad de Medellín, recorridos urbanos, e intercambios de experiencias con los talleres de diseño de las Universidades Nacional, Santo Tomás y Rémintong, bajo la orientación de los docentes titulares, de pares académicos y funcionarios de la EDU.

IMAGEN 2. Planos de análisis de los sectores la Ladera y la Calle.

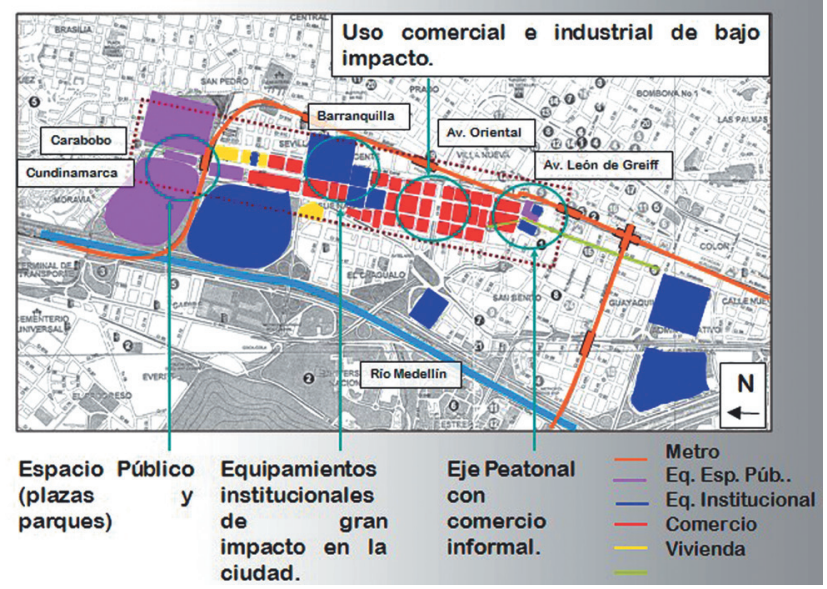

FUENTE: Grupos de trabajo Taller IX.

IMAGEN 3. Visita a Medellín, trabajo de campo
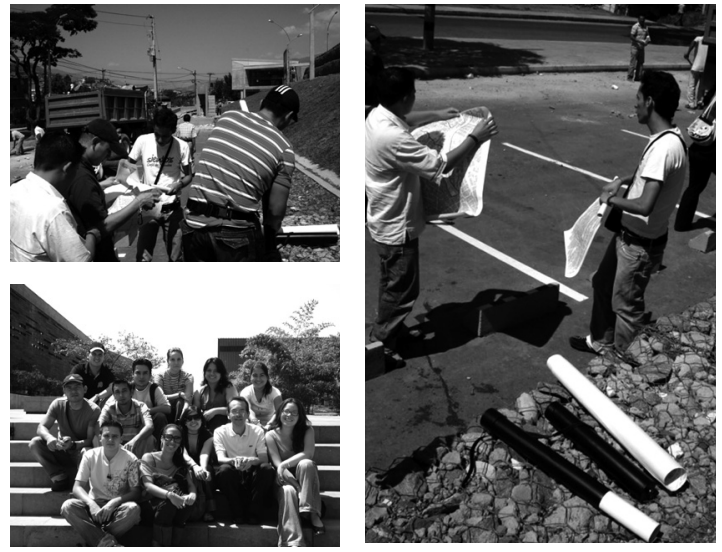

FUENTE: Grupos de trabajo Taller IX.

El desarrollo del Taller se llevó a cabo en el espacio académico regular definido y programado por la Facultad y contó con el apoyo de docentes invitados y profesionales externos, entre quienes se destacó el arquitecto Alejandro Echeverri, director de proyectos urbanos de la Alcaldía de Medellín. 
IMAGEN 4: Visita de arquitectos y urbanistas expertos como apoyo al Taller.
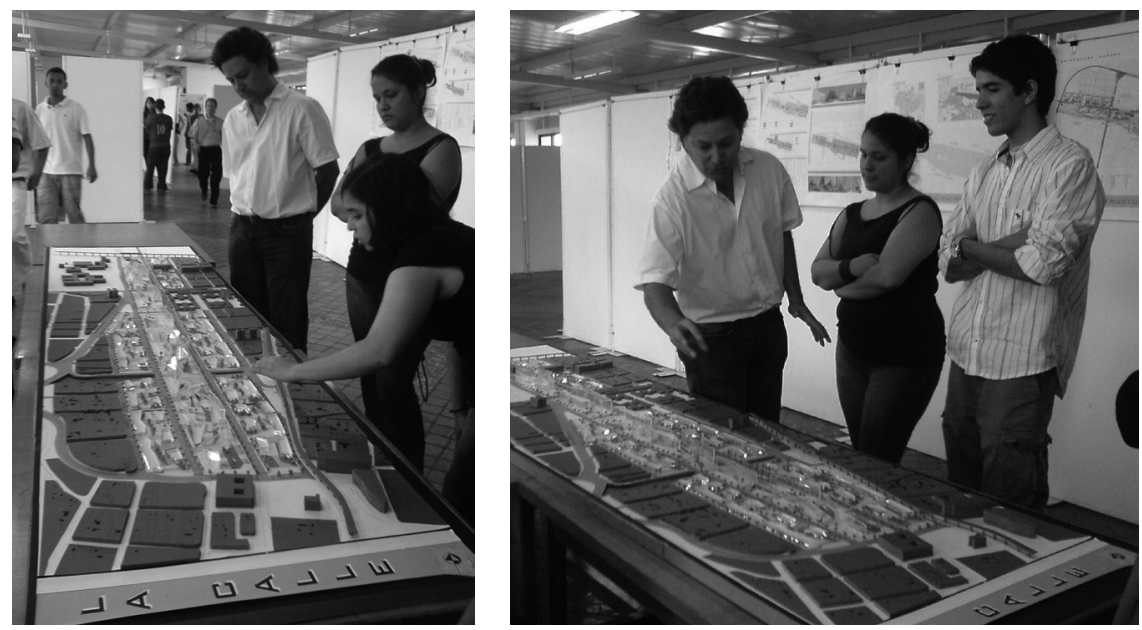

FUENTE: arquitecto José Alejandro Gómez

\section{DISCUSIÓN Y APORTES}

Bajo la premisa de la estructuración de propuestas urbanas a partir de la lectura precisa de los proyectos estratégicos desarrollados en los diferentes sectores, cada uno de los grupos de trabajo generó una serie de propuestas de recualificación urbana, entre los que se destacan los sectores de la Cárcel, la Ladera y la Calle.

\section{GRUPO No. I: SECTOR LA CÁRCEL}

- Integrantes: Edwin Jesús Balcácer García, Gustavo Enrique Rey Rueda, Gonzalo Alberto Valenzuela Bulla.

- Propuesta: Potencializar las centralidades barriales ligadas a las estaciones de Metro, establecer en la estación San Javier y Santa Lucía sistemas de espacios públicos integrados con sistemas de movilidad y con objetos de usos mixtos, a través de la conversión de la zona de la cárcel de mujeres el Buen Pastor en zona de vivienda y la consolidación de los ejes viales vehiculares, peatonales, ciclo-rutas e integrarlos con el sistema público y con las otras zonas de la ciudad.

IMAGEN 5: Plano general de la propuesta urbana e imágenes de las áreas de vivienda.

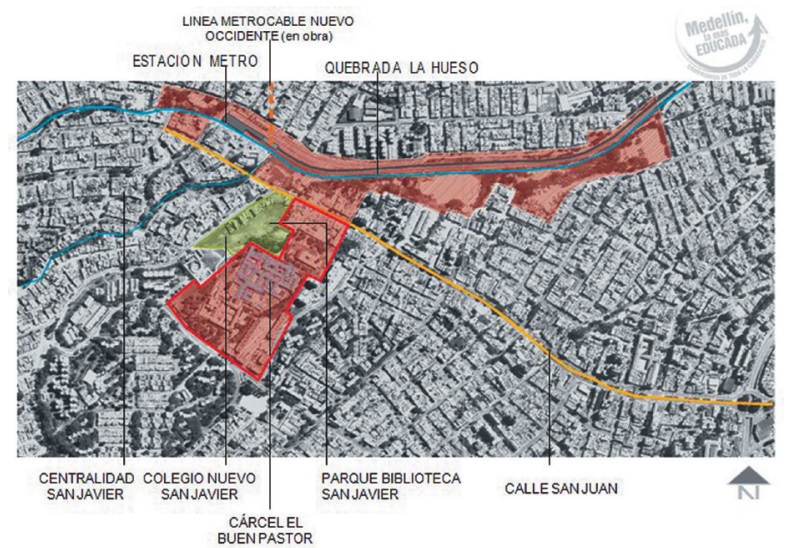

FUENTE: grupo de trabajo Taller IX. 


\section{GRUPO No. 3: SECTOR LA LADERA}

- Integrantes: Natalia Andrea Parra Torres, Gustavo Guillermo Lizcano Bermúdez, Ómar Fidel Méndez, Carlos Andrés Valencia Lopera, Jénnifer Lucía Martínez Ruiz.

- Propuesta: Generación de una alternativa de integración urbana a través de la consolidación de las áreas de equipamientos a manera de puerta urbana y conformación de proyectos de vivienda reforzada con el mejoramiento de las áreas educativas existentes y complementada con parques lineales, miradores urbanos y zonas de recreación pasiva que se extienden hacia las principales vías del entorno inmediato.

\section{GRUPO No. 4: SECTOR LA CALLE}

- Integrantes: Isabel C. Quiñones Camargo, María Bibiana Fuentes Villabona, Jenny Paola Correa Rincón, Pedro A. Ardila, Nicolás A. Romero Glen.

- Propuesta: Desarrollo de una propuesta de renovación urbana en el área central, a partir de la implementación de proyectos de comercio organizado en edificaciones adecuadas, vivienda en altura, generación de espacio público adecuado a las intensidades de uso del sector y mejoramiento de la conexión de los equipamientos existentes y propuestos.

IMAGEN 6: Plano general de la propuesta urbana.

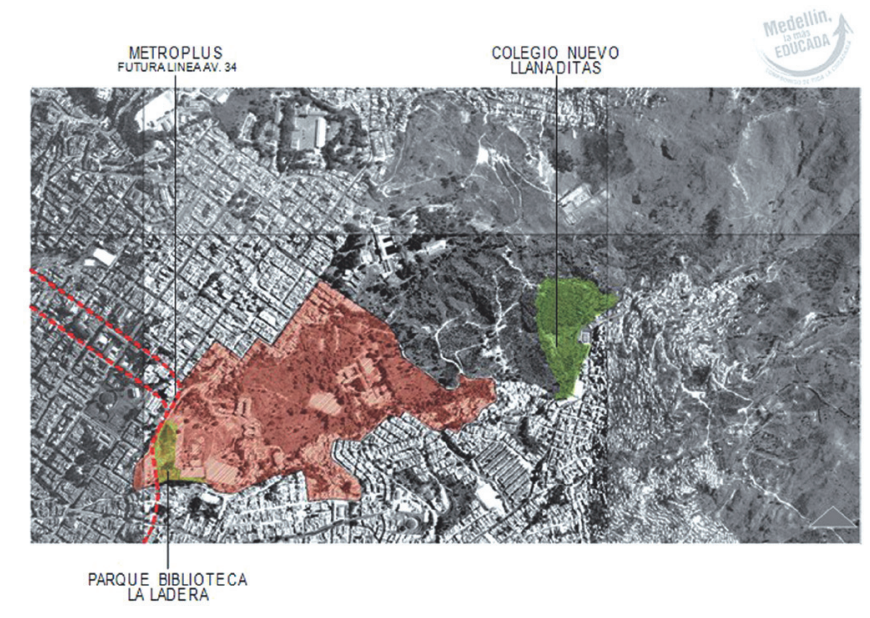

FUENTE: Grupo de trabajo Taller IX.

\section{CONCLUSIONES}

A partir del ejercicio de intervención urbano arquitectónica desarrollado en el Taller IX durante el primer semestre de 2007 en ciertos sectores de la ciudad de Medellín, la Facultad de Arquitectura abrió un espacio de interacción de doble vía: por un lado logró generar un reconocimiento de la actividad académica desarrollada en el ámbito regional y proyectarla en el contexto nacional $y$, por el otro, experimentó una forma de acercamiento a la realidad cotidiana de la ciudad y a las más recientes formas de gestión y planificación territorial desarrolladas en el país. 
Desde la perspectiva académica, se estableció un método de trabajo para el abordaje de estudios de sectores de ciudad, a partir de los proyectos estratégicos implementados como punto de partida para el desarrollo de intervenciones integrales. Así mismo, y con la estructuración de un esquema rigurosamente planificado, se logró la conformación de equipos de trabajo, que mediante la definición y asignación específica de tareas, permitió la generación de una importante base de datos, a partir de la recolección, clasificación, análisis e interpretación de la información primaria y secundaria obtenida durante el desarrollo del taller.

Paralelamente se generó un interesante debate entre docentes y alumnos, en torno a la posibilidad de recualificación de sectores urbanos en proceso de degradación física y social; $y$, sobre la aparente dualidad que imponen las actuales formas del planeamiento territorial y la intervención urbana: renovación frente a conservación, concentración frente a dispersión, multifuncionalidad frente a especialización, segregación frente a integración. Con lo anterior, se evidenció la urgencia de aunar esfuerzos entre la administración pública, el sector privado, la comunidad y, por supuesto, la universidad, con el fin de generar un mejor modelo de intervención urbana, lo que de manera ejemplar ha permitido que la ciudad de Medellín logre un alto impacto en el desarrollo de sus procesos de revitalización urbana.

\section{BIBLIOGRAFÍA}

ECHEVERRI RESTREPO, Alejandro. Medellín Problemas y Oportunidades, EDU, 2007.

. Encuentro Medellín, EDU, 2007.

HALL, Peter. Ciudades del mañana: historia del urbanismo en el Siglo XX, "La ciudad de la Belle Epoque: las infociudades y los ghettos sin información: Nueva York, Londres y Tokio 1985 - 2010", Editorial SERBAL, Barcelona, 1996.

MATUS, Carlos, Estrategia y Plan, Editorial Siglo XXI, Méjico, I98I.

OSTROWETSKY, Silvia. Artículo Las Centros Urbanos, www.memoria.com.mx,

PRADILLA, Cobos, E. (1998). Metrópolis y Megalópolis en América Latina, Revista interamericana de Planificación Nos. I19, 120.

TROITIÑO, Miguel Ángel. Revista Perspectivas Urbanas, Artículo Renovación urbana: dinámicas y cambios funcionales, Colegio de Arquitectos de Aragón, 2000.

. Artículo renovación urbana: dinámicas y cambios funcionales, Universidad

Politécnica de Cataluña, www.etsav.upc.es. 2003. 